\title{
Review of Trends in State Estimation of Power Distribution Networks
}

\author{
Jiawei Zhu, Bhuvana Ramachandran \\ Department of Electrical and Computer Engineering, University of West Florida, Pensacola, FL, USA \\ Email: zj4@students.uwf.edu, br@uwf.edu
}

How to cite this paper: Zhu, J.W. and Ramachandran, B. (2020) Review of Trends in State Estimation of Power Distribution Networks. Journal of Power and Energy Engineering, 8, 85-99.

https://doi.org/10.4236/jpee.2020.88007

Received: June 23, 2020

Accepted: August 22, 2020

Published: August 25, 2020

Copyright $\odot 2020$ by author(s) and Scientific Research Publishing Inc. This work is licensed under the Creative Commons Attribution International License (CC BY 4.0).

http://creativecommons.org/licenses/by/4.0/

\begin{abstract}
Distribution network state estimation provided complete and reliable information for the distribution management system (DMS) and was a prerequisite for other advanced management and control applications in the power distribution network. This paper first introduced the basic principles of the state estimation algorithm and sorted out the research status of the distribution network state estimation from least squares, gross error resistance etc. Finally, this paper summarized the key problems faced by the high-dimensional multi-power flow active distribution network state estimation and discussed prospects for future research hotspots and developments.
\end{abstract}

\section{Keywords}

Distribution Network, State Estimation, Weighted Least Square

\section{Introduction}

Against the backdrop of increasingly severe environmental problems and severe energy crisis, distributed generation (DG) has provided more and more alternatives to traditional energy sources in countries around the world that are actively adjusting the structure of the energy industry due to its advantages of cleanliness, low carbon and low cost. Hence more and more DGs are introduced into the distribution network due to their very many benefits. The distribution network containing distributed power generation is also called active distribution network in academic research. Adding distributed power generation sources to the distribution network can reduce the power loss caused by long-distance power transmission, and can improve the power supply reliability and quality of the power distribution system to a certain extent, enhance the voltage support of the network, and reduce the loss of the distribution network. However, after the 
DGs are connected to the distribution network, the distribution network changes from a single power supply system to a multi-power supply system. Problems such as bidirectional current and voltage cross-limits may occur on the feeder, DG power output fluctuates and the load changes randomly, all of which bring about serious challenges and many technical difficulties to the operation of the distribution network.

With the proposal and development of the concept of smart grid, a smart distribution network has also been put on the agenda of the development direction of the grid. It can be seen from the future smart distribution network plan ideas proposed by various large energy companies and research institutions, that effective energy management and control is a necessary link, and the center of management and control infrastructure is the distribution management system. Distribution management system (DMS) is an integrated application that supports decision-making systems. It can assist dispatching rooms and field operators to monitor and effectively control the distribution network in real time, enabling safe, efficient and reliable operation of the distribution network. DMS can effectively implement the network topology analysis, switch operation schedule and safety management, state estimation, network power flow analysis, voltage-reactive power control, load dump application, fault management and system recovery, load balancing through feeder reconstruction, distribution load forecasting and other applications.

As the basic core part of building DMS, distribution system state estimation (DSSE) uses real-time measurement data and load pseudo-measurement data collected by supervisory control and data acquisition (SCADA) system to calculate the comprehensive and reliable state of the distribution network through certain data processing calculations, providing reliable, high-precision, and complete real-time data for other advanced DMS application software, such as voltage regulation control and DG power output control. DSSE facilitates studying and estimating the state of the active distribution network in a safe and reliable manner for the distribution network to have a reliable and efficient operation.

State estimation of the distribution network has been relatively fruitful since the 1980s and has laid the foundation for the construction of intelligent distribution networks (active distribution networks, microgrids). Current mainstream distribution network state estimation methods mainly include weighted least squares (WLS) state estimation, robust state estimation, and state estimation of computational intelligence (particle swarm optimization, ant colony algorithm, etc.), among which WLS-based state estimation methods are the most widely used. According to the different divisions of the required state variables, the WLS estimation method includes algorithms that use node voltage and branch current as state quantities. As the most basic state estimation method, WLS has high estimation quality and good convergence, but its gross error (measurement error, malicious bad data injection, etc.) processing ability is poor, and the above gross error will inevitably affect the state estimation. In the actual distribution network, data errors are inevitable. The main sources include errors, poor ob- 
servation patterns, and poor distribution patterns. How to improve the ability of the state estimation algorithm to deal with the gross error when the gross error is obvious and reduce the impact of bad data on the estimation result as much as possible, so as to obtain the best estimated value, is basic idea of robust state estimation method.

This paper mainly introduces the development process of distribution network state estimation and classifies the solution methodologies proposed in literature using different state estimation techniques under different scenarios. Finally, future research direction and possible challenges in distribution network state estimation after transition to a Smart Grid are discussed to provide reference for future research.

The paper is structured as follows: Section 2 describes the main problems in the active distribution network state estimation, Section 3 introduces WLS state estimation method, one uses node voltage as state variable, another uses branch current, Section 4 outlines robust state estimation, Section 5 studies the state estimation of active distribution network (ADN) with distributed generation (DG). Finally, conclusions are drawn in Section 6.

\section{Problems in the Active Distribution Network State Estimation}

Power grid state estimation began in the 1960s and 1970s. Since its development, the technology has been quite mature, and the practical application of state estimation is very extensive. In the world's major power systems, energy management systems (EMS) based on state estimation are equipped to ensure the safe, reliable and economic operation of the transmission grid. However, the distribution network state estimation is estimated to have gained importance in the late $20^{\text {th }}$ century (1980-90s). The technology is not yet mature, and this is mainly because there are many differences between the distribution network and the transmission network state estimation. Table 1 lists the differences between transmission and distribution networks.

From the comparison of different features of transmission grid and distribution network, the problems of DSSE can be summarized into three aspects: 1) Detailed modeling of distribution network. 2) Less real-time measurement, more pseudo-load measurement, but low reliability. 3) Suitable state estimation algorithm for distribution network.

1) Detailed modeling of distribution network: From the analysis of the differences between the distribution network and the transmission network, the transmission network state estimation can be conducted as a single-phase analysis due to the three-phase parameter balance, but the distribution network cannot be treated as a single phase equivalent. In reference [1], a detailed analysis of the distribution network in made and the line impedance parameters are expressed in the form of a three-phase full matrix, which can model the distribution line in more detail and accuracy. The various voltage regulators (VR) in the distribution network, such as capacitor bank, on-line tap change transform (OTC) and 
Table 1. Differences between transmission and distribution networks.

\begin{tabular}{|c|c|c|}
\hline Items & Transmission Network & Distribution Network \\
\hline Topology & $\begin{array}{l}\text { The general network topology is } \\
\text { ring-shaped and needs to be } \\
\text { analyzed as a whole. }\end{array}$ & $\begin{array}{l}\text { The power supply situation is } \\
\text { regional power supply, the } \\
\text { network topology in the region } \\
\text { is radial, the closed-loop design } \\
\text { between the regions, and the } \\
\text { open-loop operation. }\end{array}$ \\
\hline $\begin{array}{l}\text { Network } \\
\text { imbalance }\end{array}$ & $\begin{array}{l}\text { The imbalance of the network is small } \\
\text { and can basically be ignored. It can be } \\
\text { considered that the three-phase line } \\
\text { parameter balance and three-phase load } \\
\text { balance can be analyzed in single-phase } \\
\text { or positive sequence. }\end{array}$ & $\begin{array}{l}\text { The three-phase line parameters } \\
\text { are unbalanced, the R/X ratio } \\
\text { fluctuates greatly, the three-phase } \\
\text { load is unbalanced, and there are } \\
\text { single-phase and two-phase loads, } \\
\text { which cannot be analyzed } \\
\text { independently. }\end{array}$ \\
\hline $\begin{array}{l}\text { SCADA } \\
\text { measuring } \\
\text { device }\end{array}$ & $\begin{array}{l}\text { There are a large number of real-time } \\
\text { measurement devices and a small } \\
\text { number of pseudo-measurements, } \\
\text { and the measurement } \\
\text { redundancy is high }\end{array}$ & $\begin{array}{l}\text { A small amount of real-time } \\
\text { measurement, a large number } \\
\text { of load pseudo-measurement, } \\
\text { from the perspective of } \\
\text { real-time measurement, the } \\
\text { measurement redundancy is low, } \\
\text { and the network value is } \\
\text { generally unobservable. }\end{array}$ \\
\hline $\begin{array}{l}\text { Network } \\
\text { scale }\end{array}$ & $\begin{array}{l}\text { A typical network generally contains } \\
\text { hundreds of buses to one or } \\
\text { two thousand buses. }\end{array}$ & $\begin{array}{l}\text { A typical network generally } \\
\text { contains } 10,000 \text { to } 100,000 \text { nodes. }\end{array}$ \\
\hline $\begin{array}{l}\text { Existing } \\
\text { power plant }\end{array}$ & $\begin{array}{l}\text { Generally, thermal power, large-scale } \\
\text { hydropower and nuclear power } \\
\text { generation, the output power is basically } \\
\text { stable and adjustable with the load. }\end{array}$ & $\begin{array}{l}\text { Mostly distributed DG distributed } \\
\text { in the feeder of the distribution } \\
\text { network, the output power } \\
\text { fluctuates greatly, and has } \\
\text { certain controllability. }\end{array}$ \\
\hline
\end{tabular}

step-type voltage regulator (STVR) exist because VR plays an important role in maintaining the grid voltage within a safe and reasonable range. Therefore, detailed distribution network modeling is the basis and premise for distribution network state estimation and other application analysis.

2) The measurement information is insufficient: Due to historical reasons for the development of the distribution network, there are only few measurement configurations and low redundancy. Under the condition that there is little real-time measurement information, load pseudo measurement plays an important role, but the existence of a large number of load pseudo measurements also directly affects the reliability of the state estimation results. While using load pseudo measurements, another important issue is the weight assigned to each load pseudo measurement. In the existing research, a uniform weight is set for all the pseudo load measurements (such as an error of $30 \%$ and a weight of 100). This unified setting method ignores the individual characteristics of the load itself and lacks certain rationality. Therefore, accurately modeling the loads, looking for the individual characteristics of the load itself, and making up for the lack of 
real-time measurements to the greatest extent, are key factors to improving the state estimation of distribution networks.

3) State estimation algorithm: The difference between distribution networks and transmission networks has caused the traditional node method and branch method to face many problems. The network structure is asymmetric, the parameters are not decoupled, the amount of calculation has surged, there are few real-time measurements, and many pseudo-measurements result in low reliability. The observability of the network is reduced, non-convergence may occur during the operation, the reliability of the estimation results is low, and there are DG sources with large power fluctuations with huge uncertainties. Therefore, it is necessary to propose an adaptive distribution network state estimation algorithm according to the network structure and measurement characteristics of the distribution network, which can handle the imbalance characteristics of the distribution network very well and better use the limited number of real-time measurements.

\section{Weighted Least Squares Estimation}

State estimation is the process of using measurements to calculate unknown parameters. Due to redundant measurements, the estimated state can be obtained by minimizing the following weighted least squares performance index $J$.

$$
J=W[z-h(x)]^{\mathrm{T}} R^{-1}[z-h(x)]
$$

The classical WLS state estimation method uses node voltage or branch current as state variables.

\subsection{Node Voltage as State Variable}

State estimation using node voltage as a state variable was first used in transmission grids. It has good estimation quality and convergence characteristics, but it requires a long calculation time and takes up a lot of memory. In view of this feature, reference [2] puts forward a fast decomposition method state estimation, ignoring the phase angle difference based on WLS. This method makes use of a matrix $\mathrm{H}$, thereby accelerating the convergence speed and reducing the memory usage. However, in the general distribution network, the feeders and loads are three-phase unbalanced and untransposed, so three-phase models must be adopted to analyze the branch flow. Reference [3] [4] [5] established a three-phase model for distribution network state estimation and used the magnitude and phase angle of the bus voltage as state variables. Observations found that the measurement functions are more complicated than the single-phase case because they contain not only the product term between adjacent nodes, but also the interphase cross-phase. In order to make the equation easier to iterate and solve, the reference [6] [7] introduced rotation variables, that is, two and three phases of moving voltage $120^{\circ}$ and $-120^{\circ}$. Since the transformation eliminates the $120^{\circ}$ angle difference between the phases, the new variables simplify the measurement 
equation. In addition, reference [8] proposed a measurement transformation technique, whose basic idea is to convert various measurements into equivalent complex current measurements. By deducing the corresponding state variables of the equivalent complex current measurement, the elements in $\mathrm{H}$ matrix can be derived as constants related to the line parameters.

\subsection{Branch Current as State Variable}

The traditional state estimation with node voltage as the state variable has been extended to three-phase analysis as shown in section 3.1. This method can handle feeders with different topologies, but the complexity is higher. In the actual distribution network, in most cases the feeder is radial, so a method tailored for distribution network state estimation was developed. In 1995, Mesut E. Baran and Arthur W. Kelley proposed a three-phase state estimation method based on branch current [9]. This method can solve the problem that arises due to radial or weak loop feeders that will produce circulating current when closing some normally open switches. The specific method is to use the branch current as a state variable to convert the power measurement to an equivalent complex current measurement. Reference [10] [11] performs special processing on the current amplitude measurement, so that the current amplitude measurement corresponds to the constant of the $\mathrm{H}$ matrix element. For the situation where voltage amplitude measurement cannot be processed, reference [12] proposes a voltage measurement processing algorithm based on phase decoupling, which uses the branch current to represent the bus voltage. Experiments show that this method can effectively handle voltage measurement and can obtain more accurate results, which can lay the foundation for the development of other applications and research based on branch current formula.

\section{Estimation of Robust State of Distribution Network}

As the basis of state estimation algorithm, the WLS method has the advantages of high estimation accuracy, easy convergence, simple model, and suitable for multiple measurements. Especially for data with normal distribution, WLS can achieve unbiased estimation. However, in the distribution network, due to the instantaneous failure of the communication line, the intermittent failure of the instrument, the use of low-precision pseudo measurement, and so on, occasional so-called gross errors (the data seriously deviate from the actual) occurs. WLS is very sensitive to gross errors. When the data cannot follow normal distribution, the least square method will lose the original good characteristics, and even a bad data may cause the estimation results to deviate from the actual. In the past, in the face of influence of bad data, the traditional method of state estimation is generally to establish a special monitoring section of bad data and put the identification of bad data after the state estimation. However, it turns out that these methods cannot handle gross errors well, so robust state estimation is the need of the hour. 


\subsection{Classic Method of Robust State Estimation}

The robust state of the distribution network is estimated to start late. Huber proposed the concept of $\mathrm{M}$ estimation for the first time in [13]. Its original motivation was to pursue the stability of parameter estimation, while least square estimation lacked stability in the face of gross error. However, stability is not equal to optimality. For data that satisfies the normal distribution, if the stability is considered too much, the efficiency of the algorithm will be reduced. Reference [14] introduced $M$ estimation into the power system for the first time in the form of a non-quadratic criterion, and proposed a bad data suppression (BDS) state estimation based on a penalty function, where the penalty function is a combination of least squares and least square root weighting. To some extent, the algorithm assigns less weight to the larger elements in the residual, so it is less sensitive to bad data. The disadvantage of $\mathrm{M}$ estimation is that it is difficult to handle more leverage measurements in the distribution network.

In order to improve the anti-interference ability of the state estimation, the common idea is to improve the structural robustness of the estimator, that is, to increase the spatial information of the system structure in the estimator. The method includes Mallows type GM estimation [15] and Schweppe type GM estimation [16]. Reference [15] introduces a weight function in the objective function, the purpose of which is to reduce the weight of the lever measurement, but such a processing method is not good for the lever measurement of good data, so there is a certain irrationality. Reference [16] proposed to expand the definition domain in state estimation, to achieve the purpose of increasing the spatial information of the system structure. This method can handle one bad lever measurement and improve the crash pollution rate, but it is difficult to handle multiple bad lever measurements. In addition, the classic $\mathrm{M}$ estimation method also includes LAV, WLAV, QC, QL, etc. [17] [18]. In the field of distribution network state estimation, the most active research is WLAV estimation. Reference [18] shows that WLAV estimation can detect and eliminate bad data at the same time by interpolating actual measurement values without any measurement filtering technology, and WLAV technology can also be incorporated into the tracking state estimator to solve the continuous measurement existing in newer problems, so WLAV estimation can be used to replace traditional WLS estimation.

\subsection{New Method of Robust State Estimation}

For improving the anti-interference of the algorithm, the existing approach is to change the weight or the objective function, so that the algorithm has a certain resistance. Reference [19] selects the appropriate variable weight function according to the principle of equivalent weight and establishes an exponential weight function WLAV state estimation (EFWLAV) of the three-phase distribution network with equality constraints. The traditional WLAV algorithm will have severe weight function oscillation or slow convergence when the residual 
error is close to zero, so the objective function model is established:

$$
J(x)=\sum_{i=1}^{m}\left(\frac{w_{i}}{\mathrm{e}^{\left|r_{i}\right|}}\right) r_{i}^{2}=\sum_{i=1}^{m}\left[\rho\left(r_{i}\right) r_{i}^{2}\right]
$$

In the formula, $X$ is the state variable. $w_{i}$ is the weight. $r_{i}$ is the residual. The improved model has properties similar to least squares for normal measurement and has good convergence performance. For bad data, the weight function is sensitive to residual changes and has good convergence properties. Reference [20] [21] proposed the state estimation method (MES) of the exponential objective function, and established a continuously differentiable state estimation model of the objective function:

$$
\left\{\begin{array}{l}
\max J(x)=\sum_{i=1}^{m} w_{i} \exp \left\{\frac{-\left[z_{i}-h_{i}(x)\right]^{2}}{2 \sigma^{2}}\right\} \\
\text { s.t. } c(x)=0
\end{array}\right.
$$

In the formula, $x$ is the state variable. $h_{i}(x)$ is the measurement function. $Z_{i}$ is the measurement. $\sigma$ is the width of the Parzen window. $c(x)=0$ is the equality constraint. The test proves that MES can automatically remove bad data without adding additional bad data monitoring links. Compared with traditional GM estimation, it can handle bad leverage measurement better, so it has a good application prospect. Different from the selection of traditional state variables, reference [22] proposes to use the square of the first-end power of the branch and the amplitude of the branch currents as the state variables on the basis of MES state estimation. This method considers the characteristics of a large amount of current amplitude measurement in the distribution network. There are three state variables in each branch, combined with the equal constraints of each branch, so it does not affect the observability of the system. The advantage of this processing method is that it does not require measurement conversion for the feeder that mainly measures current amplitude and is suitable for the radial or weak ring structure of the distribution network. It is a tailor-made for the distribution network methods. In addition, unlike the common three-phase three-wire system model, reference [23] proposed the SE model of the three-phase four-wire system of the distribution network, and used an average clustering algorithm to identify bad data, effectively solving the poor convergence problem.

The MES mentioned in this section can be equivalent to the state estimation under the definition of Renyi quadratic entropy. The current common robust state estimation also includes the new information graph state estimation, the most favorable state estimation, the maximum pass rate state estimation, etc. Among them, reference [24] applied the new information graph theory to the power system, which can identify the topology errors in the measurement system and better identify the bad data in the state estimation. The method proposed by [25] no longer measures the distance between the measured value and the estimated value but measures the approval status at most. This method can handle bad data and leverage measurement well, but the calculation efficiency is 
low. Reference [26] proposed to convert the state estimation problem into nonlinear programming, since it greatly improves the calculation efficiency of the algorithm and has certain applications in the state estimation of distribution networks.

\section{Distribution Network with Distributed Power State Estimation}

In recent years, in order to cope with the national sustainable development strategy, clean energy such as solar energy has become a research hotspot. With the rapid development of these DG and energy storage components, on the one hand, the shortage of power resources has been effectively alleviated, and the problem of environmental pollution has been reduced. On the other hand, the grid connection of DG has also resulted in higher requirement of safety and stability of the power system. Especially in the case of distribution networks that are directly connected to users, their safe and stable operation is very important.

With the access of various DG and energy storage systems, the operation, monitoring and control of the distribution network have become more and more complicated, and is mainly reflected in: the access of high permeability DG and active load makes the traditional distribution network into an active distribution network, the power flow direction is no longer unidirectional, and the uncertain network model makes the traditional distribution network state estimation method no longer applicable. The randomness and volatility of DG are greater, and the changes are more complicated, requiring state estimation cycle to be shorter. The use of smart measurement instruments provides more accurate and reliable real-time measurement data, but traditional state estimation cannot meet the requirements of massive data in terms of processing speed and accuracy.

State estimation is an indispensable part of the intelligent distribution system. This section mainly studies the state estimation of ADN with DG, introduces common model algorithms and means to improve the estimation accuracy, and discusses its research hotspots.

\subsection{Modeling of New Pseudo-Measurements in Active Distribution Networks}

Compared with traditional distribution network state estimation, the biggest difference of active distribution network state estimation is distributed power modeling. Reference [27] [28] established a single-phase distribution network state estimation model for DG access. Reference [29] established a three-phase unbalanced model, treating DG as an injection node for power. Reference [30] assumed three-phase power equal, according to the historical data of the distributed power output to get a pseudo measurement of power of each phase. Although these processing methods are simple, they have great limitations in the three-phase unbalanced distribution network connected by DGs. In this regard, reference [31] proposed a distributed three-phase state estimation of active dis- 
tribution network with multiple types of DG, established a model of DG direct grid connection and DG grid connection via PWM converter, and expanded the state variables. On the AC side,

$$
x_{\mathrm{AC}}=\left[x E_{0} \delta_{0}\right]^{\mathrm{T}}
$$

(where $x$ is the node voltage state variable, $E_{0}$ and $\delta_{0}$ are the states of the distributed power supply DG). Finally, according to the different forms of grid connection, the method of obtaining pseudo-measurement in different forms is given to ensure the observability of the system.

The integration of intermittent power generation, unpredictable demand and limited measurement increases the uncertainty of the distribution network, and the deterministic model of the distribution network leads to wrong solution of the actual system operating state. In order to avoid such situations, generally system variables are represented randomly. Random and probabilistic studies in power systems can determine the most likely voltage conditions on any bus, as well as the probability of voltage and power flow exceeding allowable operating limits. Reference [32] studied the role of wind power and other input variables in distribution network power flow research and state estimation. The Gaussian mixture method was used to accurately model any probability density and the probability density function (PDF) was uniformly expressed:

$$
f(z)=\sum_{i^{\prime}=1}^{N_{c}} w_{i^{\prime}} f_{i^{\prime}}\left(z \mid \mu_{i^{\prime}}, \sigma_{i^{\prime}}^{2}\right)
$$

In the formula, $N_{c}$ is the total number of uncertain variables. $f_{i^{\prime}}\left(z \mid \mu_{i^{\prime}}, \sigma_{i^{\prime}}^{2}\right)$ is the probability density function of the uncertain quantity. $\mu_{i^{\prime}}, \sigma_{i^{\prime}}^{2}$ are standard deviation and variance respectively.

The variance of Equation (4) is expressed and brought into the traditional WLS estimation, and the state variables of the system are iterated again. This method gives a set of related random input variables, which can determine the random power flow and bus voltage within a specific time of interest. The results obtained can be used for real-time measurement and monitoring.

\subsection{Improvement of State Estimation Algorithm}

The presence of DG leads to an increase in the dimension of the distribution network and the difficulty of solving the state estimation. While remodeling the active distribution network, it is also necessary to improve the traditional state estimation algorithm. Common algorithm improvements mainly focus on error representation, fast solution strategies, and linearized state estimation algorithms. In the process of solving the traditional state estimation, the inverse of the information matrix has no real physical meaning, and the influence of different measurement information on the estimation result cannot be seen. In this regard, reference [33] proposed a method for expressing the state estimation error with a circuit, observe the information matrix:

$$
D=H^{\mathrm{T}} W^{-1} H=\left(d_{i j}\right)_{m \times n}
$$




$$
d_{i j}=\sum_{k^{\prime}=1}^{m} h_{k^{\prime}}\left(\sum_{l=1}^{m} \frac{h_{k^{\prime} j}}{w_{k^{\prime} l}}\right)
$$

In the formula, $k^{\prime} l$ are the quantity sequence number. $i$ and $j$ are the node sequence numbers. The information matrix is a symmetric matrix whose elements are easy to find. According to the measurement of the elements and the system, and the regularity of the network topology, the corresponding correlation state and correlation strength are obtained, thereby reflecting the physical electrical distance and the measurement error. This algorithm can more intuitively show the influence of measurement accuracy on state estimation results than traditional algorithms.

The phasor measurement units (PMU) can obtain more accurate data, so that the linear relationship between the injection measurement and the branch measurement can be used to redefine the state estimation problem. Taking advantage of PMU's ability to estimate precise phasors and inherent real-time characteristics, reference [34] proposed a three-phase linear state estimation method based on PMU, which speeds up the calculation speed. Taking the three-phase voltage of the node as the state variable, the three-phase node voltage and the branch current obtained in the PMU are measured as the quantity, and the linear relationship between the corresponding quantity measurement and the state variable can be obtained. It should be noted that when using classic power measurement or amplitude measurement, the state estimation model is nonlinear and inaccurate. Reference [34] takes state estimation as the target of realtime application, so only synchronous phasor measurement is considered, instead of using pseudo measurement.

\subsection{Optimization of Measurement Devices and Robust Configuration}

The future active distribution network is characterized by significantly and rapidly changing operating conditions, such as intermittent power injection of renewable energy. In view of its characteristics, reference [35] considers the lack of detailed information about distributed power generation in the process of optimal instrument configuration, and considers the possible non-Gaussian distribution of distributed power generation, using the so-called Gaussian component combination method, the Gaussian mixture model introduced into layout optimization. On this basis, the state expression under the mixed measurement of PMU and SM is obtained, with the lowest measurement cost as the objective function and the lowest estimated accuracy as the constraint condition, and the mathematical model for the optimization of measurement device is established and solved.

In recent years, the research on optimization of distribution network measurement devices is mostly based on a network structure. However, with the development and application of network reconstruction technology, its impact on the distribution network state estimation cannot be ignored. Reference [36] pro- 
posed a distribution network measurement configuration method that considers network reconfiguration. This method determines the saturation number of the measurement system, obtains the operating probability of each network structure through a Markov chain, and establishes the following model:

$$
\left\{\begin{array}{l}
\min E_{c}=\sum_{\gamma=1}^{N_{s t r}} p_{\gamma} E_{s \gamma} \\
\text { s.t. } m \leq N_{s a t}
\end{array}\right.
$$

In the formula, $N_{s t r}$ is the number of network structures. $E$ is the system state estimation error. $N_{s a t}$ is the saturation measurement. $p$ is the running probability of the network structure. Finally, any of the heuristic algorithm solution is used to determine the installation location of the measurement sensor.

\section{Conclusions}

In general, the research on distribution network state estimation is not mature. Considering the structural characteristics of the distribution network itself, how to apply the more mature transmission state estimation method to the threephase unbalanced distribution network is an important research direction in the future. This paper discusses the state variable selection, algorithm optimization, measurement modeling and other issues in distribution network state estimation, and uses weighted least squares estimation, robust state estimation and DG access state estimation to estimate the state of distribution networks. The characteristics of the above three types of algorithms are now summarized.

1) The weighted least square method is the basis of the state estimation of the distribution network. Its algorithm model is simple and can handle a variety of measurements. It can achieve unbiased estimation of data that meets the normal distribution, but the calculation speed is slow, and the memory consumption is large. Moreover, it cannot handle gross errors.

2) Robust state estimation is an improvement based on classical least squares estimation and has a certain ability to deal with gross errors. For a distribution network that lacks real-time measurement and requires a lot of pseudo-measurements with low accuracy, robust state estimation can more accurately estimate the real-time state of the system.

3) The state estimation of the distribution network accessed by DG is more complicated, with high dimensions, model uncertainty, multi-power flow direction, etc. The original state estimation method needs to be improved from the aspects of measurement devices, algorithms, and models. Due to sustainable development strategy, more DGs will be connected to the distribution network in the future, and the state estimation of the active distribution network accessed by DG will become the focus of research.

Considering the opportunities and challenges facing the current distribution network state estimation, the following points have important research value: intermittent power supply of distributed power supply, network reconstruction and other reasons make the study of uncertainty particularly important. There will be more uncertain factors in the study of state estimation, such as the un- 
certainty of load, DG output and network parameters. Large-scale active distribution network increases the dimension of the system and will generate massive data, traditional string centralized state estimation method will no longer be applicable, and more and faster distributed parallel state estimation methods need to be developed, which is of great significance to satisfy the real-time monitoring and control of the distribution network.

The promotion and application of intelligent measurement system has greatly increased the rate of data flow. The distribution network has obtained an unprecedented amount of data. Processing a large amount of bad data and applying advanced big data analysis methods to the distribution network state estimation will become research hotspot and a challenge in the future. All in all, the active distribution network is a key link in the construction of smart grids in the future, and it is very important to update the distribution network state estimation technology. Based on the research in recent years, the three-phase unbalanced parallel state estimation of the active distribution network based on big data analysis can be continued in the future to accelerate the modernization of the distribution network.

\section{Acknowledgements}

The authors would like to thank the Department of Electrical and Computer Engineering, University of West Florida for supporting this research project.

\section{Conflicts of Interest}

The authors declare no conflicts of interest regarding the publication of this paper.

\section{References}

[1] Kersting, W.H. (2012) Distribution System Modeling and Analysis. CRC Press, Boca Raton. https://doi.org/10.1201/b12056-31

[2] Yu, E. (1985) Power System State Estimation. Hydraulic and Electric Power Press, Beijing.

[3] Li, K. (1996) State Estimation for Power Distribution System and Measurement Impacts. IEEE Transactions on Power Systems, 11, 911-916. https://doi.org/10.1109/TPWRS.2009.2016599

[4] Hansen, C.W. and Debs, A.S. (1995) Power System State Estimation Using ThreePhase Models. IEEE Transactions on Power Systems, 10, 818-824.

https://doi.org/10.1109/59.387922

[5] Falcao, D.M., Wu, F.F. and Murphy, L. (1995) Parallel and Distributed State Estimation. IEEE Transactions on Power Systems, 10, 724-730. https://doi.org/10.1109/59.387909

[6] Wei, Z., Gu, C., Ju, P. and Gong, B. (2000) A Three-Phase Radial Distribution System State Estimation Algorithm. Proceedings of the CSEE, 20, 84-87.

[7] Baran, M.E. and Kelley, A.W. (1994) State Estimation for Real-Time Monitoring of Distribution Systems. IEEE Transactions on Power Systems, 9, 1601-1609.

https://doi.org/10.1109/59.336098 
[8] Wang, G., Giannakis, G.B. and Chen, J. (2019) Robust and Scalable Power System State Estimation via Composite Optimization. IEEE Transactions on Smart Grid, 10, 6137-6147. https://doi.org/10.1109/TSG.2019.2897100

[9] Baran, M.E. and Kelley, A.W. (1995) A Branch-Current-Based State Estimation Method for Distribution Systems. IEEE Transactions on Power Systems, 10, 483-491. https://doi.org/10.1109/59.373974

[10] Pau, M., Pegoraro, P.A., Monti, A., Muscas, C., Ponci, F. and Sulis, S. (2018) Impact of Current and Power Measurements on Distribution System State Estimation Uncertainty. IEEE Transactions on Instrumentation and Measurement, 68, 3992-4002. https://doi.org/10.1109/TIM.2018.2883844

[11] Majidi, M., Etezadi-Amoli, M. and Livani, H. (2017) Distribution System State Estimation Using Compressive Sensing. International Journal of Electrical Power \& Energy Systems, 88, 175-186. https://doi.org/10.1016/j.ijepes.2016.12.016

[12] Wang, G., Giannakis, G.B., Chen, J. and Sun, J. (2019) Distribution System State Estimation: An Overview of Recent Developments. Frontiers of Information Technology \& Electronic Engineering, 20, 4-17. https://doi.org/10.1631/FITEE.1800590

[13] Huber, P.J. (1992) Robust Estimation of a Location Parameter. In: Breakthroughs in Statistics, Springer, New York, 492-518. https://doi.org/10.1007/978-1-4612-4380-9 35

[14] Merrill, H.M. and Schweppe, F.C. (1971) Bad Data Suppression in Power System Static State Estimation. IEEE Transactions on Power Apparatus and Systems, PAS-90, 2718-2725. https://doi.org/10.1109/TPAS.1971.292925

[15] Hampel, F.R., Ronchetti, E.M., Rousseeuw, P.J. and Stahel, W.A. (2011) Robust Statistics: The Approach Based on Influence Functions (Vol. 196). John Wiley \& Sons, Hoboken.

[16] Handschin, E., Schweppe, F.C., Kohlas, J. and Fiechter, A.A.F.A. (1975) Bad Data Analysis for Power System State Estimation. IEEE Transactions on Power Apparatus and Systems, 94, 329-337. https://doi.org/10.1109/T-PAS.1975.31858

[17] Zhao, J., Wang, S., Mili, L., Amidan, B., Huang, R. and Huang, Z. (2018) A Robust State Estimation Framework considering Measurement Correlations and Imperfect Synchronization. IEEE Transactions on Power Systems, 33, 4604-4613. https://doi.org/10.1109/TPWRS.2018.2790390

[18] Lin, Y. and Abur, A. (2018) Robust State Estimation against Measurement and Network Parameter Errors. IEEE Transactions on Power Systems, 33, 4751-4759. https://doi.org/10.1109/TPWRS.2018.2794331

[19] Qin, S. (2016) The Research and Application of Robust State Estimation on Distribution Base on Exponential Function. Master's Thesis, Hunan University, Changsha.

[20] Zang, H., Geng, M., Xue, M., Mao, X., Huang, M., Chen, S., Sun, G., et al. (2019) A Robust State Estimator for Integrated Electrical and Heating Networks. IEEE Access, 7, 109990-110001. https://doi.org/10.1109/ACCESS.2019.2933525

[21] Ho, W.K., Chen, T., Ling, K.V. and Sun, L. (2017) Variance Analysis of Robust State Estimation in Power System Using Influence Function. International Journal of Electrical Power \& Energy Systems, 92, 53-62. https://doi.org/10.1016/j.ijepes.2017.04.009

[22] Zhao, J., Netto, M. and Mili, L. (2016) A Robust Iterated Extended Kalman Filter for Power System Dynamic State Estimation. IEEE Transactions on Power Systems, 32, 3205-3216. https://doi.org/10.1109/TPWRS.2016.2628344

[23] Karimipour, H. and Dinavahi, V. (2017) Robust Massively Parallel Dynamic State 
Estimation of Power Systems against Cyber-Attack. IEEE Access, 6, 2984-2995. https://doi.org/10.1109/ACCESS.2017.2786584

[24] Xing, R. and Kewen, W. (2019) Automatic Partition for Distributed and Robust State Estimation of Distribution Network. Electrical Measurement \& Instrumentation, No. 1, 12.

[25] Zheng, W., Wu, W., Shi, X., Zhang, B. and Yang, J. (2016) A Robust Bilinear ThreePhase State Estimation Method for Power Systems. 2016 IEEE Power and Energy Society General Meeting, Boston, 17-21 July 2016, 1-4. https://doi.org/10.1109/PESGM.2016.7741284

[26] Wang, X., Zhao, J., Terzija, V. and Wang, S. (2020) Fast Robust Power System Dynamic State Estimation Using Model Transformation. International Journal of Electrical Power \& Energy Systems, 114, Article ID: 105390. https://doi.org/10.1016/j.ijepes.2019.105390

[27] Ullah, Z., Elkadeem, M.R., Wang, S. and Radosavljević, J. (2020) A Novel PSOSCGSA Method for State Estimation in Unbalanced DG-Integrated Distribution Systems. IEEE Access, 8, 113219-113229. https://doi.org/10.1109/ACCESS.2020.3003521

[28] Gu, W., Lou, G., Tan, W. and Yuan, X. (2017) A Nonlinear State Estimator-Based Decentralized Secondary Voltage Control Scheme for Autonomous Microgrids. IEEE Transactions on Power Systems, 32, 4794-4804. https://doi.org/10.1109/TPWRS.2017.2676181

[29] Chen, S., Wei, Z., Sun, G., Lu, N., Sun, Y. and Zhu, Y. (2017) Multi-Area Distributed Three-Phase State Estimation for Unbalanced Active Distribution Networks. Journal of Modern Power Systems and Clean Energy, 5, 767-776. https://doi.org/10.1007/s40565-016-0237-0

[30] Dehghanpour, K., Wang, Z., Wang, J., Yuan, Y. and Bu, F. (2018) A Survey on State Estimation Techniques and Challenges in Smart Distribution Systems. IEEE Transactions on Smart Grid, 10, 2312-2322. https://doi.org/10.1109/TSG.2018.2870600

[31] Wei, Z., Chen, S., Sun, G., Wang, D. and Sun, Y. (2015) Distributed Three-Phase State Estimation for Active Distribution Network Integrated with Different Types of Distributed Generators. Automation of Electric Power Systems, 39, 68-74.

[32] Valverde, G., Saric, A.T. and Terzija, V. (2012) Stochastic Monitoring of Distribution Networks Including Correlated Input Variables. IEEE Transactions on Power Systems, 28, 246-255. https://doi.org/10.1109/TPWRS.2012.2201178

[33] Chen, X., Lin, J. and Song, Y. (2014) A Circuit Representing Method for State Estimation Errors in Distribution Networks. Proceedings of the CSEE, 34, 4839-4846.

[34] Sarri, S., Zanni, L., Popovic, M., Le Boudec, J.Y. and Paolone, M. (2016) Performance Assessment of Linear State Estimators Using Synchrophasor Measurements. IEEE Transactions on Instrumentation and Measurement, 65, 535-548. https://doi.org/10.1109/TIM.2015.2510598

[35] Liu, J., Ponci, F., Monti, A., Muscas, C., Pegoraro, P.A. and Sulis, S. (2013) Optimal Placement for Robust Distributed Measurement Systems in Active Distribution Grids. 2013 IEEE International Instrumentation and Measurement Technology Conference, Minneapolis, 6-9 May 2013, 206-211. https://doi.org/10.1109/I2MTC.2013.6555410

[36] Wang, H., Zhang, W. and Feng, Z. (2016) Robust Measurement Placement Method for Distribution Systems Considering Fault Reconfiguration. Automation of Electric Power Systems, 40, 98-104. 